PACS Numbers: 74.20.Fg, 74.25.Bt, 74.62.Dh

\title{
Effect of nonmagnetic and magnetic impurities on the specific heat jump in anisotropic superconductors
}

\author{
Leonid A. Openov \\ Moscow Engineering Physics Institute (State University) \\ 115409 Moscow, Russia \\ opn@supercon.mephi.ru
}

\begin{abstract}
The specific-heat jump $\Delta C$ at a critical temperature $T_{c}$ in an anisotropic superconductor containing both potential and spin-flip scatterers is calculated within a weak-coupling mean-field approximation. It is shown that the presence of even a small amount of spin-flip scatterers in the sample leads to a drastic change in the dependence of $\Delta C$ on $T_{c}$ in a disordered $(d+s)$-wave or a strongly anisotropic $s$-wave superconductor. The implications for experimental tests for the presence of an $s$-wave admixture in the superconducting order parameter of high- $T_{c}$ superconductors are discussed.
\end{abstract}

\section{INTRODUCTION}

Although there is a considerable evidence in favour of predominantly $d$-wave in-plane symmetry of the superconducting order parameter $\Delta(\mathbf{p})$ in high-temperature superconductors (HTSCs) [1] a number of experiments point to an admixture of the $s$-wave component to $d$-wave $\Delta(\mathbf{p})$, implying a mixture of $d$-wave and $s$-wave components, see references in Ref. 1], or even to a strongly anisotropic $s$-wave $\Delta(\mathbf{p})$, see Refs. 2, 3]. The nonpure $d$-wave symmetry of $\Delta(\mathbf{p})$ is supported in part by a long tail suppression of the critical temperature, $T_{c}$, by defects and impurities $[4,5]$ characteristic for a nonzero value of the Fermi surface (FS) average $\langle\Delta(\mathbf{p})\rangle_{F S}$, while $\langle\Delta(\mathbf{p})\rangle_{F S}=0$ for a $d$-wave pairing. However, neither the presence nor the absence of an isotropic $s$-wave component of $\Delta(\mathbf{p})$ in HTSCs has not been demonstrated unequivocally yet.

Recently Harań et al. [6] have shown that significant features attributed to the $s$-wave part of $\Delta(\mathbf{p})$ may be seen in the measurements of the specific heat jump $\Delta C$ at $T_{c}$ in disordered $(d+s)$-wave superconductors with nonmagnetic impurities. It was found in Ref. [6] that there is a qualitative difference in the evolution of $\Delta C$ under disordering in $d$-wave and $(d+s)$-wave superconductors. While in a $d$-wave superconductor, the value of $\Delta C$ normalized by the normal state specific heat $C_{n}\left(T_{c}\right)$ monotonously goes to zero as $T_{c}$ is suppressed by nonmagnetic disorder, in a $(d+s)$-wave superconductor there is a minimum at the curve of $\Delta C / C_{n}\left(T_{c}\right)$ versus $T_{c}$. The location of this minimum depends on a specific weight of an isotropic $s$-wave component in the $(d+s)$-wave $\Delta(\mathbf{p})$, i. e., on a specific value of $\langle\Delta(\mathbf{p})\rangle_{F S}$. It was suggested in Ref. [6] that this effect may be used as a test for the presence of an $s$-wave admixture in HTSCs.

The authors of Ref. [6] restricted their consideration to the case of nonmagnetic disorder (i. e., potential scattering of charge carriers) only. Note, however, that a lot of experiments give evidence for the presence of spin-flip scatterers (along with potential ones) in non-stoichiometric HTSCs, e. g., in oxygen-deficient, doped or irradiated samples (see, e. g., the references in Ref. [7). The spin-flip scattering of charge carriers by magnetic impurities (even though they may be present in small proportion) can strongly modify the value of $T_{c}$ as well as other characteristics of a superconductor in the vicinity of $T_{c}$ [7, 8]. It is therefore instructive to elucidate if an account for spin-flip scattering will change the conclusion drawn in Ref. 6] concerning the impurity effect on $\Delta C$ in anisotropic superconductors.

The influence of the ratio of spin-flip to non spin-flip scattering rates on the specific heat jump in a weakly anisotropic superconductor has been considered earlier by Okabe and Nagi [9]. In the present paper we study the combined effect of potential and spin-flip scatterers on $\Delta C$ in a superconductor with arbitrary anisotropy of $\Delta(\mathbf{p})$, including $s$-wave, $d$-wave and $(d+s)$-wave as particular cases. In what follows, we make use of the following approximations: (i) we consider the pairing interactions in the weak-coupling limit of the BCS model and assume that the pairing potential can be taken in a factorizable form; (ii) we treat the impurity scattering in the Born limit; (iii) we assume an $s$-wave scattering of charge carriers by both potential and spin-flip scatterers, so that the scattering matrix elements are momentum independent; (iv) we neglect any dynamic pair breaking effects 10 .

One should keep in mind that the mean-field BCS theory does not describe the effects of spatial variation of the order parameter in the vicinity of impurities 11. These effects may be significant in superconductors with short coherence length. However, experiments on the impurity induced suppression of $T_{c}$ in anisotropic superconductors are, in fact, rather well described within the mean-field Abrikosov-Gor'kov approach [12] (see, e. g., [13]). So, the spatial variation of the order parameter may appear to have a little influence on the physical characteristics, including $\Delta C$, in the vicinity of $T_{c}$. Note also that strong-coupling corrections [14] result just 
in renormalization of the scattering rates [15] and overall increase in the magnitude of $\Delta C$, without qualitative changes in the dependence of $\Delta C$ versus $T_{c}$ [16, 17]. As for the use of the weak scattering (Born) limit, it seems to be justified by a very close similarity of $\Delta C$ versus $T_{c}$ curves calculated in the Born and unitary limits for the case of purely nonmagnetic disorder in $d$-wave and $(d+s)$-wave superconductors [6, 16, 18, We set $\hbar=k_{B}=1$ throughout the paper.

\section{FORMALISM}

The specific heat jump is defined as $\Delta C=C_{s}\left(T_{c}\right)-C_{n}\left(T_{c}\right)$, where the subscripts $s$ and $n$ refer to the superconducting and normal state respectively, $C_{n}\left(T_{c}\right)=\left(2 \pi^{2} / 3\right) N(0) T_{c}$, and $N(0)$ is the density of electron states per spin at the Fermi level. The value of $\Delta C$ can be expressed in terms of the thermodynamic potential $\Omega$ as

$$
\Delta C=-T_{c}\left[\frac{\partial^{2}\left(\Omega_{s}-\Omega_{n}\right)}{\partial T^{2}}\right]_{T=T_{c}} .
$$

For the value of the difference $\Omega_{s}-\Omega_{n}$ one has (see, e. g., Ref. [12])

$$
\Omega_{s}-\Omega_{n}=\int_{0}^{\Delta^{2}} \frac{d\left(V_{0}^{-1}\right)}{d \Delta^{2}} \Delta^{2} d \Delta^{2}
$$

where $V_{0}$ is the pairing energy that determines the magnitude of the phenomenological factorizable pairing potential of the form $V\left(\mathbf{p}, \mathbf{p}^{\prime}\right)=-V_{0} \phi(\mathbf{n}) \phi\left(\mathbf{n}^{\prime}\right), \Delta$ is the amplitude of the superconducting order parameter $\Delta(\mathbf{p})=\Delta \phi(\mathbf{n}), \mathbf{n}=\mathbf{p} / p$ is a unit vector along the momentum, and the function $\phi(\mathbf{n})$ specifies the symmetry and anisotropy of $\Delta(\mathbf{p})$ in the momentum space [e. g., $\phi(\mathbf{n}) \equiv$ const for isotropic $s$-wave pairing; $\phi(\mathbf{n})=\cos (2 \varphi)$ for a specific case of $d$-wave pairing, where $\varphi$ is an angle between the vector $\mathbf{n}$ and the $x$-axis; $\phi(\mathbf{n})=r+\cos (2 \varphi)$ for a specific case of mixed $(d+s)$-wave pairing, the constant $r$ being the measure of the partial weight of $s$-wave component in $\Delta(\mathbf{p})]$.

To find the dependence of $V_{0}^{-1}$ on $\Delta$, we make use of the set of mean-filed self-consistent equations for a superconductor containing both nonmagnetic and magnetic impurities (see Ref. [7]):

$$
\begin{gathered}
\Delta(\mathbf{p})=-T \sum_{\omega} \sum_{\mathbf{k}} V(\mathbf{p}, \mathbf{k}) \frac{\Delta_{\omega}(\mathbf{k})}{\omega^{\prime 2}+\xi^{2}(\mathbf{k})+\left|\Delta_{\omega}(\mathbf{k})\right|^{2}} \\
\Delta_{\omega}(\mathbf{p})=\Delta(\mathbf{p})+\left(c_{n}\left|u_{n}\right|^{2}+c_{m}\left|u_{m}^{p o t}\right|^{2}-c_{m}\left|u_{m}^{e x}\right|^{2}\right) \sum_{\mathbf{k}} \frac{\Delta_{\omega}(\mathbf{k})}{\omega^{\prime 2}+\xi^{2}(\mathbf{k})+\left|\Delta_{\omega}(\mathbf{k})\right|^{2}}, \\
\omega^{\prime}=\omega-i\left(c_{n}\left|u_{n}\right|^{2}+c_{m}\left|u_{m}^{p o t}\right|^{2}+c_{m}\left|u_{m}^{e x}\right|^{2}\right) \sum_{\mathbf{k}} \frac{i \omega^{\prime}+\xi(\mathbf{k})}{\omega^{\prime 2}+\xi^{2}(\mathbf{k})+\left|\Delta_{\omega}(\mathbf{k})\right|^{2}},
\end{gathered}
$$

where $T$ is the temperature; $\xi(\mathbf{p})$ is the quasiparticle energy measured from the chemical potential; $\omega=\pi T(2 n+$ 1) are Matsubara frequencies; $c_{n}$ and $c_{m}$ are the concentrations of nonmagnetic and magnetic impurities, respectively; $u_{n}$ is the matrix element for potential electron scattering by an isolated nonmagnetic impurity; $u_{m}^{p o t}$ and $u_{m}^{e x}$ are the matrix elements for, respectively, potential and exchange (spin-flip) scattering by an isolated magnetic impurity.

Restricting all electron momenta in Eqs. (3) - (5) to the FS, replacing $\sum_{\mathbf{k}}$ by $N(0) \int d \xi(\mathbf{k}) \int_{F S} d \Omega_{\mathbf{k}} /|\partial \xi(\mathbf{k}) / \partial \mathbf{k}|$, and integrating over $\xi(\mathbf{k})$, one has

$$
\begin{gathered}
V_{0}^{-1}=\pi N(0)\left\langle\phi^{2}(\mathbf{n})\right\rangle_{F S} T \sum_{\omega} f(\omega, \Delta), \\
\Delta_{\omega}(\mathbf{p})=\Delta(\mathbf{p})+\frac{1}{2}\left(\frac{1}{\tau_{p}}-\frac{1}{\tau_{s}}\right)\left\langle\frac{\Delta_{\omega}(\mathbf{p})}{\sqrt{\omega^{\prime 2}+\left|\Delta_{\omega}(\mathbf{p})\right|^{2}}}\right\rangle_{F S}, \\
\omega^{\prime}=\omega+\frac{1}{2}\left(\frac{1}{\tau_{p}}+\frac{1}{\tau_{s}}\right) \omega^{\prime}\left\langle\frac{1}{\sqrt{\omega^{\prime 2}+\left|\Delta_{\omega}(\mathbf{p})\right|^{2}}}\right\rangle_{F S},
\end{gathered}
$$

where

$$
f(\omega, \Delta)=\frac{1}{\left\langle\phi^{2}(\mathbf{n})\right\rangle_{F S} \Delta}\left\langle\frac{\phi(\mathbf{n}) \Delta_{\omega}(\mathbf{p})}{\sqrt{\omega^{\prime 2}+\left|\Delta_{\omega}(\mathbf{p})\right|^{2}}}\right\rangle_{F S},
$$


the angular brackets $\langle\ldots\rangle_{F S}$ stand for a FS average,

$$
\langle\ldots\rangle_{F S}=\int_{F S}(\ldots) \frac{d \Omega_{\mathbf{p}}}{|\partial \xi(\mathbf{p}) / \partial \mathbf{p}|} / \int_{F S} \frac{d \Omega_{\mathbf{p}}}{|\partial \xi(\mathbf{p}) / \partial \mathbf{p}|},
$$

and we have introduced the electron relaxation times $\tau_{p}$ and $\tau_{s}$ for potential and spin-flip scattering, respectively:

$$
\frac{1}{\tau_{p}}=2 \pi\left(c_{n}\left|u_{n}\right|^{2}+c_{m}\left|u_{m}^{p o t}\right|^{2}\right) N(0), \frac{1}{\tau_{s}}=2 \pi c_{m}\left|u_{m}^{e x}\right|^{2} N(0)
$$

\section{RESULTS}

Making use of a standard procedure, one can transform Eq. (6) into

$$
\ln \left(\frac{T}{T_{c 0}}\right)=\pi T \sum_{\omega}\left(f(\omega, \Delta)-\frac{1}{|\omega|}\right),
$$

where $T_{c 0}$ is the value of $T_{c}$ in the absence of impurities. At $\Delta=0$, one obtains from Eqs. (7)-(9), (12) an expression for $T_{c}$ as a function of potential and spin-flip scattering rates, $\rho_{p}=1 / 4 \pi \tau_{p} T_{c}$ and $\rho_{s}=1 / 2 \pi \tau_{s} T_{c}$, respectively (see Ref. [7])

$$
\ln \left(\frac{T_{c 0}}{T_{c}}\right)=\frac{\langle\phi(\mathbf{n})\rangle_{F S}^{2}}{\left\langle\phi^{2}(\mathbf{n})\right\rangle_{F S}}\left[\Psi\left(\frac{1}{2}+\rho_{s}\right)-\Psi\left(\frac{1}{2}\right)\right]+\left(1-\frac{\langle\phi(\mathbf{n})\rangle_{F S}^{2}}{\left\langle\phi^{2}(\mathbf{n})\right\rangle_{F S}}\right)\left[\Psi\left(\frac{1}{2}+\rho\right)-\Psi\left(\frac{1}{2}\right)\right]
$$

where $\rho=\rho_{p}+\rho_{s} / 2$ is the total scattering rate and $\Psi$ is the digamma function.

Expanding $f(\omega, \Delta)$ in powers of $\Delta^{2}$ up to $\Delta^{2}$ and differentiating Eq. (6) with respect to $\Delta^{2}$, one has from Eq. (2) in the vicinity of $T_{c}$

$$
\Omega_{s}-\Omega_{n}=\frac{\pi}{2} N(0)\left\langle\phi^{2}(\mathbf{n})\right\rangle_{F S} \Delta^{4}(T) T \sum_{\omega}\left(\frac{d f(\omega, \Delta)}{d \Delta^{2}}\right)_{\Delta=0} .
$$

Next, taking Eq. (13) into account, one has from Eq. (12) an expression for $\Delta^{2}(T)$ in the vicinity of $T_{c}$

$$
\Delta^{2}(T)=\left(\frac{T}{T_{c}}-1\right) \frac{\left\langle\phi^{2}(\mathbf{n})\right\rangle_{F S}-\rho\left[\left\langle\phi^{2}(\mathbf{n})\right\rangle_{F S}-\langle\phi(\mathbf{n})\rangle_{F S}^{2}\right] \Psi^{(1)}\left(\frac{1}{2}+\rho\right)-\rho_{s}\langle\phi(\mathbf{n})\rangle_{F S}^{2} \Psi^{(1)}\left(\frac{1}{2}+\rho_{s}\right)}{\pi\left\langle\phi^{2}(\mathbf{n})\right\rangle_{F S} T_{c} \sum_{\omega}\left(\frac{d f(\omega, \Delta)}{d \Delta^{2}}\right)_{\Delta=0}} .
$$

Here and below $\psi^{(n)}(z)$ are the polygamma functions [the $\mathrm{n}$-th derivatives of the digamma function $\psi(z)$ ] defined as $\psi^{(n)}(z)=(-1)^{n+1} n ! \sum_{k=0}^{\infty}(k+z)^{-(n+1)}$.

Substituting Eq. (15) into Eq. (14), we obtain from Eq. (1) the expression for the specific heat jump $\Delta C$ normalized by the specific heat in the normal state,

$$
\frac{\Delta C}{C_{n}\left(T_{c}\right)}=-12 \frac{\left[\left\langle\phi^{2}(\mathbf{n})\right\rangle_{F S}-\rho\left[\left\langle\phi^{2}(\mathbf{n})\right\rangle_{F S}-\langle\phi(\mathbf{n})\rangle_{F S}^{2}\right] \Psi^{(1)}\left(\frac{1}{2}+\rho\right)-\rho_{s}\langle\phi(\mathbf{n})\rangle_{F S}^{2} \Psi^{(1)}\left(\frac{1}{2}+\rho_{s}\right)\right]^{2}}{\left(2 \pi T_{c}\right)^{3}\left\langle\phi^{2}(\mathbf{n})\right\rangle_{F S} \sum_{\omega}\left(\frac{d f(\omega, \Delta)}{d \Delta^{2}}\right)_{\Delta=0}} .
$$

Finally, after simple but rather cumbersome calculations, we find from Eqs. (7)-(9) an expression for the denominator in Eq. (16)

$$
\begin{aligned}
& \left(2 \pi T_{c}\right)^{3}\left\langle\phi^{2}(\mathbf{n})\right\rangle_{F S} \sum_{\omega}\left(\frac{d f(\omega, \Delta)}{d \Delta^{2}}\right)_{\Delta=0} \\
& =\frac{\rho}{6} \Psi^{(3)}\left(\frac{1}{2}+\rho\right)\left[\left\langle\phi^{2}(\mathbf{n})\right\rangle_{F S}-\langle\phi(\mathbf{n})\rangle_{F S}^{2}\right]^{2}+\frac{\rho_{s}}{6} \Psi^{(3)}\left(\frac{1}{2}+\rho_{s}\right)\langle\phi(\mathbf{n})\rangle_{F S}^{4}+\frac{1}{2} \Psi^{(2)}\left(\frac{1}{2}+\rho_{s}\right)\langle\phi(\mathbf{n})\rangle_{F S}^{4} \\
& +\frac{1}{2} \Psi^{(2)}\left(\frac{1}{2}+\rho\right)\left[\left\langle\phi^{4}(\mathbf{n})\right\rangle_{F S}-4\left\langle\phi^{3}(\mathbf{n})\right\rangle_{F S}\langle\phi(\mathbf{n})\rangle_{F S}+6\left\langle\phi^{2}(\mathbf{n})\right\rangle_{F S}\langle\phi(\mathbf{n})\rangle_{F S}^{2}-3\langle\phi(\mathbf{n})\rangle_{F S}^{4}\right] \\
& +\frac{1}{\rho-\rho_{s}} \Psi^{(1)}\left(\frac{1}{2}+\rho\right)\left[4\left\langle\phi^{3}(\mathbf{n})\right\rangle_{F S}\langle\phi(\mathbf{n})\rangle_{F S}-11\left\langle\phi^{2}(\mathbf{n})\right\rangle_{F S}\langle\phi(\mathbf{n})\rangle_{F S}^{2}+7\langle\phi(\mathbf{n})\rangle_{F S}^{4}\right] \\
& -\frac{5}{\rho-\rho_{s}} \Psi^{(1)}\left(\frac{1}{2}+\rho_{s}\right)\langle\phi(\mathbf{n})\rangle_{F S}^{2}\left[\left\langle\phi^{2}(\mathbf{n})\right\rangle_{F S}-\langle\phi(\mathbf{n})\rangle_{F S}^{2}\right]
\end{aligned}
$$




$$
\begin{aligned}
& +\frac{\rho+\rho_{s}}{\left(\rho-\rho_{s}\right)^{2}}\left[\Psi^{(1)}\left(\frac{1}{2}+\rho\right)+\Psi^{(1)}\left(\frac{1}{2}+\rho_{s}\right)\right]\langle\phi(\mathbf{n})\rangle_{F S}^{2}\left[\left\langle\phi^{2}(\mathbf{n})\right\rangle_{F S}-\langle\phi(\mathbf{n})\rangle_{F S}^{2}\right] \\
& -\frac{4}{\left(\rho-\rho_{s}\right)^{2}}\left[\Psi\left(\frac{1}{2}+\rho\right)-\Psi\left(\frac{1}{2}+\rho_{s}\right)\right]\left[\left\langle\phi^{3}(\mathbf{n})\right\rangle_{F S}\langle\phi(\mathbf{n})\rangle_{F S}-4\left\langle\phi^{2}(\mathbf{n})\right\rangle_{F S}\langle\phi(\mathbf{n})\rangle_{F S}^{2}+3\langle\phi(\mathbf{n})\rangle_{F S}^{4}\right] \\
& -2 \frac{\rho+\rho_{s}}{\left(\rho-\rho_{s}\right)^{3}}\left[\Psi\left(\frac{1}{2}+\rho\right)-\Psi\left(\frac{1}{2}+\rho_{s}\right)\right]\langle\phi(\mathbf{n})\rangle_{F S}^{2}\left[\left\langle\phi^{2}(\mathbf{n})\right\rangle_{F S}-\langle\phi(\mathbf{n})\rangle_{F S}^{2}\right] .
\end{aligned}
$$

Eqs. (16) and (17), together with Eq. (13) for $T_{c}$, give the value of the specific heat jump for a superconductor that is characterized by an arbitrary anisotropy of $\Delta(\mathbf{p})$ [i. e., by an arbitrary angular function $\phi(\mathbf{n})]$ and contains, in general, both potential and spin-flip scatterers. In particular cases of (i) spin-flip scattering in an isotropic $s$-wave superconductor with $\phi(\mathbf{n}) \equiv$ const and (ii) potential scattering in a highly anisotropic (e. g., $d$-wave) superconductor with $\langle\phi(\mathbf{n})\rangle_{F S}=0$, Eqs. (16) and (17) reduce to the well-known expressions 6. 18, 19, 20, 21]

$$
\frac{\Delta C}{C_{n}\left(T_{c}\right)}=-12 \frac{\left[1-\rho_{s} \Psi^{(1)}\left(\frac{1}{2}+\rho_{s}\right)\right]^{2}}{\frac{1}{2} \Psi^{(2)}\left(\frac{1}{2}+\rho_{s}\right)+\frac{\rho_{s}}{6} \Psi^{(3)}\left(\frac{1}{2}+\rho_{s}\right)}
$$

and

$$
\frac{\Delta C}{C_{n}\left(T_{c}\right)}=-12 \frac{\left[1-\rho \Psi^{(1)}\left(\frac{1}{2}+\rho\right)\right]^{2}}{\frac{1}{2} \frac{\left\langle\phi^{4}(\mathbf{n})\right\rangle_{F S}}{\left\langle\phi^{2}(\mathbf{n})\right\rangle_{F S}^{2}} \Psi^{(2)}\left(\frac{1}{2}+\rho\right)+\frac{\rho}{6} \Psi^{(3)}\left(\frac{1}{2}+\rho\right)},
$$

respectively 22]. In the case of a superconductor that has an arbitrary anisotropy of $\Delta(\mathbf{p})$ but contains nonmagnetic impurities only, Eqs. (16) and (17) reduce to the results of Harań et al. [6, 22]. In the absence of any impurities $\left(T_{c}=T_{c 0}\right)$, one has

$$
\frac{\Delta C}{C_{n}\left(T_{c 0}\right)}=\frac{12}{7 \zeta(3)} \frac{\left\langle\phi^{2}(\mathbf{n})\right\rangle_{F S}^{2}}{\left\langle\phi^{4}(\mathbf{n})\right\rangle_{F S}}
$$

where $\zeta(3) \approx 1.202$ is the Riemann zeta function. Eq. (20) has been widely used to analyze the effect of anisotropy of $\Delta(\mathbf{p})$ on the specific heat jump in clean superconductors (see, e. g., [23]). For a clean isotropic $[\phi(\mathbf{n}) \equiv$ const $]$ superconductor we arrive at a familiar BCS result, $\Delta C / C_{n}\left(T_{c 0}\right)=12 / 7 \zeta(3) \approx 1.426$.

\section{DISCUSSION}

In what follows, we shall model the dependence of $\Delta(\mathbf{p})=\Delta \phi(\mathbf{n})$ by the angular function $\phi(\mathbf{n})=r+$ $\cos (2 \varphi)$. The value of $r=0$ corresponds to $d$-wave pairing, while $r \rightarrow \infty(\Delta r \rightarrow$ const $)$ in an isotropic $s$-wave superconductor. The smaller is the value of $r$, the higher is the anisotropy of $\Delta(\mathbf{p})$. The moments $\langle\phi(\mathbf{n})\rangle_{F S}$, $\left\langle\phi^{2}(\mathbf{n})\right\rangle_{F S},\left\langle\phi^{3}(\mathbf{n})\right\rangle_{F S}$, and $\left\langle\phi^{4}(\mathbf{n})\right\rangle_{F S}$ that enter Eqs. (13), (16), and (17) are equal to $r, r^{2}+1 / 2, r^{3}+3 r / 2$, and $r^{4}+3 r^{2}+3 / 8$, respectively.

Note that the value of the specific heat jump in a clean superconductor is a nonmonotonous function of $r$. It follows from Eq. (20) that the normalized specific heat jump initially decreases with $r$ from $\Delta C / C_{n}\left(T_{c 0}\right) \approx 0.951$ at $r=0$ down to $\Delta C / C_{n}\left(T_{c 0}\right) \approx 0.666$ at $r=\sqrt{3 / 8} \approx 0.612$ and next increases again up to $\Delta C / C_{n}\left(T_{c 0}\right)=$ $12 / 7 \zeta(3) \approx 1.426$ at $r \rightarrow \infty$, see Fig. 1 .

Now let us analyze the behavior of $\Delta C / C_{n}\left(T_{c}\right)$ versus $T_{c}$ upon addition of magnetic and/or nonmagnetic impurities to the initially clean sample with the critical temperature $T_{c 0}$. We note that nonmagnetic impurities result in the potential scattering only, while magnetic impurities generally result in both spin-flip and potential scattering. In this respect, the combined effect of nonmagnetic and magnetic impurities has much in common with the effect of magnetic impurities only, the difference being in the ratio of potential to spin-flip scattering rates as a function of impurity concentrations.

\section{A. Nonmagnetic disorder}

First, we consider the case that there are no magnetic impurities in the sample, i. e., $\rho_{s}=0$ and, hence, $\rho=\rho_{p}$ (see also Ref. [6]). At low concentration of nonmagnetic impurities, i. e., at $\left(T_{c 0}-T_{c}\right) / T_{c 0}<<1$, one has from Eqs. (16) and (17)

$$
\frac{\Delta C}{C_{n}\left(T_{c}\right)}=\frac{12}{7 \zeta(3)} \frac{\left\langle\phi^{2}(\mathbf{n})\right\rangle_{F S}^{2}}{\left\langle\phi^{4}(\mathbf{n})\right\rangle_{F S}}\left[1-2 \frac{T_{c 0}-T_{c}}{T_{c 0}}\left(1+\frac{\pi^{2}}{42 \zeta(3)} \cdot \frac{\left\langle\phi^{2}(\mathbf{n})\right\rangle_{F S}}{\left\langle\phi^{4}(\mathbf{n})\right\rangle_{F S}} \cdot \frac{4\left\langle\phi^{3}(\mathbf{n})\right\rangle_{F S}\langle\phi(\mathbf{n})\rangle_{F S}-3\left\langle\phi^{4}(\mathbf{n})\right\rangle_{F S}-\left\langle\phi^{2}(\mathbf{n})\right\rangle_{F S}^{2}}{\left\langle\phi^{2}(\mathbf{n})\right\rangle_{F S}-\langle\phi(\mathbf{n})\rangle_{F S}^{2}}\right)\right],
$$

where we took into account that $\rho=\left(2 / \pi^{2}\right)\left(1-T_{c} / T_{c 0}\right)\left\langle\phi^{2}(\mathbf{n})\right\rangle_{F S} /\left[\left\langle\phi^{2}(\mathbf{n})\right\rangle_{F S}-\langle\phi(\mathbf{n})\rangle_{F S}^{2}\right]$ at $\left(T_{c 0}-T_{c}\right) / T_{c 0}<<1$, see Eq. (13) and Ref. [7. Note that the term in round brackets in Eq. (21) changes sign from positive to negative 
as $r$ increases up to $r_{0} \approx 1.75$. Analysis of Eqs. (16), (17), and (21) shows that in weakly anisotropic superconductors with $r>r_{0}$, the normalized specific heat jump increases monotonously up to $\Delta C / C_{n}\left(T_{c}\right)=12 / 7 \zeta(3)$ as $T_{c}$ is suppressed by nonmagnetic impurities, in a close agreement with the behaviour of $\Delta C / C_{n}\left(T_{c}\right)$ in two-gap superconductors whose thermodynamics is, to some respect, similar to that of anisotropic superconductors, see Ref. 24] (note that in an isotopic superconductor, $r \rightarrow \infty$, the nonmagnetic disorder has no effect on both $T_{c}$ and $\Delta C / C_{n}\left(T_{c}\right)$, see Refs. [12, 25]). Contrary, at $0<r<r_{0}$, the normalized specific heat jump initially decreases with decreasing $T_{c}$, passes through a minimum at $T_{c} / T_{c 0}=\left(T_{c} / T_{c 0}\right)^{*}$, and then increases up to $\Delta C / C_{n}\left(T_{c}\right)=12 / 7 \zeta(3)$ as $T_{c} \rightarrow 0$. The position of the minimum at the curve of $\Delta C / C_{n}\left(T_{c}\right)$ versus $T_{c} / T_{c 0}$ depends on $r$, the value of $\left(T_{c} / T_{c 0}\right)^{*}$ being reduced from 1 to 0 as $r$ decreases from $r_{0}$ down to zero [6].

In a $d$-wave superconductor without an admixture of $s$-wave, i. e., at $r=0$, the normalized specific heat jump decreases monotonously down to zero as $T_{c} / T_{c 0}$ is suppressed from 1 to 0 . For an arbitrary function $\phi(\mathbf{n})$ obeying the condition $\langle\phi(\mathbf{n})\rangle_{F S}=0$ we have at $T_{c} / T_{c 0}<<1$

$$
\frac{\Delta C}{C_{n}\left(T_{c}\right)}=\frac{8\left\langle\phi^{2}(\mathbf{n})\right\rangle_{F S}^{2}}{3\left\langle\phi^{4}(\mathbf{n})\right\rangle_{F S}-2\left\langle\phi^{2}(\mathbf{n})\right\rangle_{F S}^{2}} \gamma^{2}\left(\frac{T_{c}}{T_{c 0}}\right)^{2},
$$

where $\gamma=e^{C} \approx 1.781$ and $C$ is the Euler constant. Note that $\left\langle\phi^{4}(\mathbf{n})\right\rangle_{F S}>\left\langle\phi^{2}(\mathbf{n})\right\rangle_{F S}^{2}$ for any $\phi(\mathbf{n})$, so the denominator in Eq. (22) is always positive. For our choice of $\phi(\mathbf{n})=r+\cos (2 \varphi)$ one has $\Delta C / C_{n}\left(T_{c}\right)=$ $(16 / 5) \gamma^{2}\left(T_{c} / T_{c 0}\right)^{2}$ at $r=0$ and $T_{c}<<T_{c 0}$.

The effect of nonmagnetic impurities on the specific heat jump in superconductors with different anisotropy of the order parameter is illustrated in Fig. 2. The difference in the behavior of $\Delta C / C_{n}\left(T_{c}\right)$ at $T_{c} \rightarrow 0$ in superconductors with $r=0$ and $r \neq 0$ stems from the fact that, while $T_{c}$ of a $d$-wave superconductor vanishes at a finite value of $\rho_{p}=1 / 4 \gamma \approx 0.140$ (i. e., at a finite concentration of nonmagnetic impurities), the value of $T_{c}$ in a superconductor with the nonzero Fermi surface average of $\Delta(\mathbf{p})$ asymptotically goes to zero as $\rho_{p}$ increases at $\rho_{s}=0$, see Refs. [7, 26].

\section{B. Pure spin-flip disorder}

Although pure spin-flip scattering never happens in real materials since magnetic impurities give rise to not only spin-flip scattering but to potential scattering as well, we nevertheless (partly for pedagogical purposes) consider briefly the limiting case that a superconductor contains spin-flip scatterers only, i. e., $\rho_{p}=0$ and $\rho=\rho_{s} / 2$. It is straightforward to show that in this case there are no qualitative differences among the curves of $\Delta C / C_{n}\left(T_{c}\right)$ versus $T_{c} / T_{c 0}$ for different values of $r$. From Eqs. (16) and (17) one finds that the normalized specific heat jump decreases monotonously down to zero with decreasing $T_{c}$, no matter what the symmetry and the degree of anisotropy of $\Delta(\mathbf{p})$ are, see Fig. 3. The physical reason is that spin-flip scatterers, contrary to potential ones, are pair breakers in both $d$-wave and $s$-wave superconductors [7]. Two quantitative differences between $d$-wave and $s$-wave symmetries are (i) the different values of $\Delta C / C_{n}\left(T_{c}\right)$ at $T_{c}=T_{c 0}$ and (ii) the different values of the coefficient $f[\phi(\mathbf{n})]$ in the dependence $\Delta C / C_{n}\left(T_{c}\right)=f[\phi(\mathbf{n})]\left(T_{c} / T_{c 0}\right)^{2}$ at $T_{c} / T_{c 0}<<1$. For a $d$-wave superconductor, the value of $f[\phi(\mathbf{n})]$ coincides with that in the case of nonmagnetic disorder, see Eq. (22), while $f=8 \gamma^{2}$ for an isotropic $s$-wave superconductor, in agreement with Ref. [12].

\section{Combined nonmagnetic and magnetic disorder}

\section{Constant concentration of spin-flip scatterers}

Now we turn to a general case that there are both nonmagnetic and magnetic impurities in a superconductor. To begin with, we consider a situation when purely nonmagnetic impurities are added to a superconductor that already contains a small quantity of magnetic impurities and, as a consequence, initially has the critical temperature $T_{c 0}^{\prime}$ lower than the value of $T_{c 0}$ in the absence of any impurities. The higher is the concentration of magnetic impurities, $c_{m}$, the greater is the value of $\delta T_{c o} / T_{c o}$, where $\delta T_{c o}=T_{c 0}-T_{c 0}^{\prime}$. We assume that the value of $c_{m}$ remains unchanged upon increase in the concentration of nonmagnetic impurities and corresponding decrease of $T_{c}$, i. e., $\rho_{s 0}=1 / 2 \pi \tau_{s} T_{c 0}=$ const. Since our prime interest here is with the case $\delta T_{c o} / T_{c o}<<1$, we plot $\Delta C / C_{n}\left(T_{c}\right)$ versus $T_{c} / T_{c 0}$ rather than $T_{c} / T_{c 0}^{\prime}$.

In a $d$-wave superconductor, i. e., at $r=0$, the dependence of $\Delta C / C_{n}\left(T_{c}\right)$ on $T_{c} / T_{c 0}$ for any value of $\delta T_{c o} / T_{c o}$ is the same as in the absence of spin-flip scattering, see Fig. 2. This is because at $\langle\phi(\mathbf{n})\rangle_{F S}=0$ both $T_{c}$ and $\Delta C$ are functions of the total scattering rate $\rho=\rho_{p}+\rho_{s} / 2$ only, irrespective of the scatterers' type, see Eqs. (13), (16), and (17). Fig. 4 shows the curves of $\Delta C / C_{n}\left(T_{c}\right)$ versus $T_{c} / T_{c 0}$ for different values of $r \neq 0$ and $\delta T_{c 0} / T_{c 0}$. At low $r=0.2$, i. e., in a $(d+s)$-wave superconductor with a small admixture of $s$-wave, or in a strongly anisotropic $s$-wave superconductor, the presence of finite, though very small $\left(\delta T_{c 0} / T_{c 0}<0.01\right)$ amount of spin-flip scatterers in the sample results in a drastic change in the dependence of $\Delta C / C_{n}\left(T_{c}\right)$ on 
$T_{c} / T_{c 0}$. After passing through a minimum, $\Delta C / C_{n}\left(T_{c}\right)$ does not increase up to $12 / 7 \zeta(3)$, as in the absence of magnetic impurities, but reaches the maximum and then decreases again down to zero as $T_{c} \rightarrow 0$, see Fig. 4 . It is straightforward to show from Eqs. (13), (16), and (17) that

$$
\frac{\Delta C}{C_{n}\left(T_{c}\right)}=\frac{1}{2 \rho_{s 0}^{2}}\left(\frac{T_{c}}{T_{c 0}}\right)^{2}=\frac{\pi^{4}}{32}\left[1+\frac{\langle\phi(\mathbf{n})\rangle_{F S}^{2}}{\left\langle\phi^{2}(\mathbf{n})\right\rangle_{F S}}\right]^{2}\left(\frac{T_{c}}{\delta T_{c 0}}\right)^{2}
$$

at $T_{c}<<\delta T_{c 0}$. As the concentration of spin-flip scatterers (and, hence, the value of $\delta T_{c 0} / T_{c 0}$ ) increases, the maximum of $\Delta C / C_{n}\left(T_{c}\right)$ decreases in height and gradually disappears. Starting with $\delta T_{c 0} / T_{c 0} \approx 0.02$ there are neither minimum nor maximum of $\Delta C / C_{n}\left(T_{c}\right)$ versus $T_{c} / T_{c 0}$ curve. We note that for $T_{c 0} \approx 100 \mathrm{~K}$ such values of $\delta T_{c 0} / T_{c 0}$ correspond to commonly observed small (several Kelvins) variations of $T_{c 0}$ between the samples obtained under slightly different conditions that can reflect the different amount of magnetic impurities in the samples.

As $r$ increases, i. e., as the anisotropy of $\Delta$ (p) becomes weaker, the tendency in the change of the specific heat jump upon increase in the initial concentration of magnetic impurities is qualitatively conserved, see Fig. 4. Quantitatively, an increase in $r$ results in the increase in the value of $\delta T_{c 0} / T_{c 0}$ above which $\Delta C / C_{n}\left(T_{c}\right)$ becomes a featureless monotonous function of $T_{c} / T_{c 0}$. In particular, both the minimum and the maximum of $\Delta C / C_{n}\left(T_{c}\right)$ versus $T_{c} / T_{c 0}$ curve disappear at $\delta T_{c 0} / T_{c 0} \approx 0.1,0.25$, and 0.4 for $r=0.6,1$, and 2 , respectively. So, the sensitivity of the specific heat jump to magnetic impurities is higher in strongly anisotropic superconductors with small but nonzero values of $r$.

\section{Constant ratio of spin-flip to potential scattering rates}

Let us now consider the case that the relative contribution from spin-flip scattering to the total scattering rate, $\rho=\rho_{p}+\rho_{s} / 2$, remains constant upon disordering, i. e., the value of the dimensionless coefficient

$$
\alpha=\frac{\tau_{s}^{-1}}{\tau_{p}^{-1}+\tau_{s}^{-1}}=\frac{\left|u_{m}^{e x}\right|^{2}}{\left(c_{n} / c_{m}\right)\left|u_{n}\right|^{2}+\left|u_{m}^{p o t}\right|^{2}+\left|u_{m}^{e x}\right|^{2}}=\frac{\rho_{s} / 2}{\rho_{p}+\rho_{s} / 2},
$$

see Eq. (11), does not change upon addition of magnetic (and, in general, nonmagnetic) impurities. This holds, first, if a superconductor is doped by magnetic impurities only (i. e., $c_{n}=0$ ) and, second, if the ratio of nonmagnetic to magnetic impurity concentrations, $c_{n} / c_{m}$, remains unchanged, see Eq. (24). The latter is a reasonable approximation for doping by given chemical elements or irradiation by a given type of particles, at least at relatively low (but sufficient to destroy the superconductivity) doping levels or radiation doses.

Thus for a given degree of $\Delta(\mathbf{p})$ anisotropy (i. e., in our model, for a given value of $r$ ), the dependence of $\Delta C / C_{n}\left(T_{c}\right)$ on $T_{c} / T_{c 0}$ is governed by the value of material-dependent and disorder-dependent coefficient $\alpha$. The greater is the relative contribution from exchange scattering by magnetic impurities to the total scattering rate, the higher is the value of $\alpha$. In general, $\alpha$ ranges from 0 in the absence of exchange scattering to 1 in the absence of potential scattering. Note, however, that since there always exist two channels of carrier scattering by magnetic impurities (potential and spin-flip ones), see Eq. (11), the value of $\alpha$ is less than unity even at $c_{n}=0$. Below we consider the case $\alpha<<1$ that seems to be relevant to the experimental situation.

It follows from Eqs. (13), (16), and (17) that in a $d$-wave superconductor with $r=0$ the curves of $\Delta C / C_{n}\left(T_{c}\right)$ versus $T_{c} / T_{c 0}$ are the same for any value of $\alpha$ in the whole range of $\alpha$, see Fig. 2 and Fig. 3. Contrary, at $r \neq 0$ the specific heat jump appears to be extremely sensitive to spin-flip scattering of charge carriers. Fig. 5a shows the dependencies of $\Delta C / C_{n}\left(T_{c}\right)$ on $T_{c} / T_{c 0}$ in a strongly anisotropic non $d$-wave superconductor with $r=0.2$ for different values of $\alpha$. One can see that increase in $\alpha$ results in a gradual disappearance of the minimum (and maximum) of $\Delta C / C_{n}\left(T_{c}\right)$. In the presence of even a minor spin-flip component in the scattering potential, $\alpha \approx 0.02$, the normalized specific heat jump decreases monotonously as $T_{c}$ decreases from $T_{c 0}$ down to zero, and the curve of $\Delta C / C_{n}\left(T_{c}\right)$ versus $T_{c} / T_{c 0}$ looks like that in a d-wave superconductor. As the gap anisotropy weakens (i. e., the value of $r$ increases) the "critical"value of $\alpha$ above which the normalized specific heat jump starts to decrease monotonously under disordering first increases up to $\approx 0.05$ at $r \approx 1$ and next decreases again, see Fig.5.

\section{Implications for the experiment}

Numerous experiments on various superconductors, including borocarbides $\mathrm{Y}_{1-x} \mathrm{R}_{x} \mathrm{Ni}_{2} \mathrm{~B}_{2} \mathrm{C}(\mathrm{R}=\mathrm{Gd}$, Dy, Ho, Er) [17, organic compound (TMTSF $)_{2} \mathrm{ClO}_{4}$ [27], $\mathrm{U}_{1-x} \mathrm{Th}_{x} \mathrm{Be}_{3}$ [28], $\mathrm{HTSCs} \mathrm{YBa}_{2}\left(\mathrm{Cu}_{1-x} \mathrm{M}_{x}\right)_{3} \mathrm{O}_{7-\delta}(\mathrm{M}=$ $\mathrm{Zn}$ [29, 30, 31, 32, $\mathrm{Fe}$ [30, $\mathrm{Ni}$ 31], $\mathrm{Cr}$ [32]) and $\mathrm{La}_{1.85} \mathrm{Sr}_{0.15} \mathrm{Cu}_{1-y} \mathrm{Zn}_{y} \mathrm{O}_{4}$ 33], etc have revealed that the value of $\Delta C / C_{n}\left(T_{c}\right)$ decreases monotonously as $T_{c}$ is suppressed by impurities. To the best of our knowledge, there were no experimental indications for the nonmonotonous behaviour of $\Delta C / C_{n}\left(T_{c}\right)$ in disordered superconductors. Note, however, that the chemical substitution results not only in the suppression of $T_{c}$ and decrease of 
$\Delta C / C_{n}\left(T_{c}\right)$ but also in a very strong broadening of the superconducting transition. As a consequence, the specific heat anomaly is rapidly smeared out by the disorder, so that the dependence of $\Delta C / C_{n}\left(T_{c}\right)$ on $T_{c} / T_{c 0}$ can be determined more or less reliably, in the best case, at $T_{c} / T_{c 0}>0.3 \div 0.4$ only. Meanwhile, it follows from the results presented above that the value of $T_{c} / T_{c 0}$ below which $\Delta C / C_{n}\left(T_{c}\right)$ starts to increase under disordering depends on the degree of $\Delta(\mathbf{p})$ anisotropy and is very small in strongly anisotropic superconductors.

Recently Zhao has fitted $\Delta(\mathbf{p})$ to single-particle tunneling and angle-resolved photoemission spectra of $\mathrm{YBa}_{2} \mathrm{Cu}_{3} \mathrm{O}_{7-\delta}$ 2]. To compare his fit with our model form of $\Delta(\mathbf{p})$, it is convenient to introduce the coefficient $\chi=1-\langle\Delta(\mathbf{p})\rangle_{F S}^{2} /\left\langle\Delta^{2}(\mathbf{p})\right\rangle_{F S}$ as a measure of the degree of in-plane anisotropy of $\Delta(\mathbf{p})$, where $\langle\ldots\rangle_{F S}$ means the Fermi surface average. The range $0 \leq \chi \leq 1$ covers the cases of isotropic $s$-wave $(\Delta(\mathbf{p})=$ const, $\chi=0)$, $d$-wave $\left(\langle\Delta(\mathbf{p})\rangle_{F S}=0, \chi=1\right)$, and mixed $(d+s)$-wave or anisotropic $s$-wave $(0<\chi<1)$ symmetries of $\Delta(\mathbf{p})$. Making use of the results presented in Ref.[2], one has $\chi \approx 0.9$ for $\mathrm{YBa}_{2} \mathrm{Cu}_{3} \mathrm{O}_{7-\delta}$. Since $\chi=1 /\left(1+2 r^{2}\right)$ for $\Delta(\mathbf{p})=\Delta[r+\cos (2 \varphi)]$, this value of $\chi$ corresponds to $r \approx 0.2$. We note that the choice of $\chi \approx 0.9$ allows for a quantitative explanation [34] of the quasilinear decrease of $T_{c}$ in electron-irradiated $\mathrm{YBa}_{2} \mathrm{Cu}_{3} \mathrm{O}_{7-\delta}$ single crystals [35. As follows from Figs. 4 and 5 , at $r=0.2$, the upturn of $\Delta C / C_{n}\left(T_{c}\right)$ takes place at $T_{c} / T_{c 0} \approx 0.1$, either in the absence or at a very low concentration of spin-flip scatterers.

So, the necessary condition for the $\Delta C / C_{n}\left(T_{c}\right)$ upturn at low $T_{c} / T_{c 0}$ is, except for the non-pure $d$-wave symmetry of $\Delta(\mathbf{p})$, a relatively small contribution of spin-flip scattering to the total scattering rate. Besides, a superconductor should be disordered very uniformly so that the transition width $\Delta T_{c}$ remained lower than $T_{c}$ down to as low as possible $T_{c}$ values, in order to preserve a clear specific heat anomaly at $T_{c}$ and to make possible the experimental determination of the $\Delta C / C_{n}\left(T_{c}\right)$ versus $T_{c} / T_{c 0}$ curve in a wide region of $T_{c} / T_{c 0}$ values. In this respect, the irradiation-induced disorder has advantages over the chemical substitution. For example, in a recent paper [35], Rullier-Albenque et al. reported the results of experimental studies of $T_{c}$ degradation under electron irradiation of underdoped and optimally doped $\mathrm{YBa}_{2} \mathrm{Cu}_{3} \mathrm{O}_{7-\delta}$ single crystals. The authors of Ref. [35] succeeded in creation of an extremely uniform distribution of radiation defects over the sample, so that the value of $\Delta T_{c}$ never exceeded $5 \mathrm{~K}$. Moreover, the value of $\Delta T_{c}$ did not increase monotonously with radiation dose but had a maximum at $T_{c} / T_{c 0} \approx 0.3$ and next decreased down to $\Delta T_{c}<1 \mathrm{~K}$ at the highest dose for which the resistive superconducting transition still was observed at $T_{c} \approx 1 \mathrm{~K}$. According to the theoretical fit 34 to the experimental data [35, at $\chi=0.9$, the value of $\alpha=0.01 \pm 0.01$ is low enough for the upturn of $\Delta C / C_{n}\left(T_{c}\right)$ be observable at $T_{c} / T_{c 0} \approx 0.1$, see Fig.5. Hence, it is of great interest to study the behavior of $\Delta C$ versus $T_{c} / T_{c 0}$ in such samples.

Finally, we note that the experimentally observed nonuniversality of $\Delta C / C_{n}\left(T_{c}\right)$ versus $T_{c} / T_{c 0}$ curve that has been previously ascribed to the carrier concentration effects 31 may in fact be (at least partly) due to different contributions of spin-flip scattering to pair breaking in different superconducting materials and/or for different doping elements. We note also that it would be very interesting to study experimentally the behavior of $\Delta C / C_{n}\left(T_{c}\right)$ versus $T_{c} / T_{c 0}$ in non-cuprate superconductors with different degree of the superconducting gap anisotropy and various ratios of spin-flip to potential scattering rates.

\section{CONCLUSIONS}

We have shown that in a pure $d$-wave superconductor, the normalized specific heat jump $\Delta C / C_{n}\left(T_{c}\right)$ decreases monotonously upon disordering by both nonmagnetic and magnetic defects or impurities. So, in $d$ wave superconductors, $\Delta C / C_{n}\left(T_{c}\right)$ is, to a first approximation (keeping in mind the assumptions made), a universal function of $T_{c} / T_{c 0}$, i. e., it does not depend on the relative contribution of spin-flip scattering to the total scattering rate and, hence, on a specific type of defects and impurities. On the other hand, under nonmagnetic disordering of a superconductor with a nonzero Fermi surface average of the order parameter, $\Delta C / C_{n}\left(T_{c}\right)$ initially decreases with decreasing $T_{c}$, passes through a minimum and then increases again. The minimum at the curve of $\Delta C / C_{n}\left(T_{c}\right)$ versus $T_{c} / T_{c 0}$ moves to higher values of $T_{c} / T_{c 0}$ as the anisotropy of the order parameter becomes weaker.

In disordered strongly anisotropic non $d$-wave superconductors, $\Delta C / C_{n}\left(T_{c}\right)$ is extremely sensitive to spinflip scattering of charge carriers. At relatively weak spin-flip scattering, $\Delta C / C_{n}\left(T_{c}\right)$ becomes a featureless monotonous function of $T_{c} / T_{c 0}$. So, the spin-flip scattering of charge carriers removes the qualitative difference between the dependencies of $\Delta C / C_{n}\left(T_{c}\right)$ versus $T_{c} / T_{c 0}$ in disordered $d$-wave and $(d+s)$-wave (or anisotropic $s$-wave) superconductors. Hence, it would be very difficult to discriminate between pure $d$-wave and non pure $d$-wave $\Delta(\mathbf{p})$ if the concentration of spin-flip scatterers in the sample is higher than a certain critical value. So, to observe the nonmonotonous dependence of $\Delta C / C_{n}\left(T_{c}\right)$ on $T_{c} / T_{c 0}$ in anisotropic superconductors, one should (i) make use of uniformly disordered samples with a clearly pronounced specific heat anomaly at $T_{c}$ down to low $T_{c} / T_{c 0}$ values and (ii) minimize the concentration of the spin-flip scatterers in the sample.

\section{ACKNOWLEDGMENTS}


Discussions with R. Kishore and I. A. Semenihin are greatly acknowledged. The work was supported in part by the Russian Department of Industry, Science, and Technology under Grant No 40.012.1.1.1357.

\section{Список литературы}

[1] J. Annett, N. Goldenfeld, and A. J. Leggett, in Physical Properties of High Temperature Superconductors, edited by D. M. Ginsberg (World Scientific, Singapore, 1996), Vol.5.

[2] G.M. Zhao, Phys. Rev. B 64, 024503 (2001); cond-mat/0305483.

[3] B. H. Brandow, Phys. Rev. B 65, 054503 (2002).

[4] J. M. Valles, Jr., A. E. White, K. T. Short, R. C. Dynes, J. P. Garno, A. F. J. Levi, M. Anzlowar, and K. Baldwin, Phys. Rev. B 39, 11599 (1989).

[5] A. G. Sun, L. M. Paulius, D. A. Gajewski, M. B. Maple, and R. C. Dynes, Phys. Rev. B 50, 3266 (1994).

[6] G. Harań, J. Taylor, and A. D. S. Nagi, Phys. Rev. B 55, 11778 (1997).

[7] L. A. Openov, Phys. Rev. B 58, 9468 (1998); Pis'ma Zh. Éksp. Teor. Fiz. 66, 627 (1997) [JETP Lett. 66, $661(1997)]$.

[8] L. A. Openov, I. A. Semenihin, and R. Kishore, Phys. Rev. B 64, 012513 (2001).

[9] Y. Okabe and A. D. S. Nagi, Phys. Rev. B 28, 1323 (1983).

[10] Comparison of the dynamic pair breaking theory with available experimental results gives evidence in favour of static pair breaking effect on $\Delta C$, see P. J. Williams, Phys. Rev. B 47, 15145 (1993).

[11] M. Franz, C. Kallin, A. J. Berlinsky, and M. I. Salkola, Phys. Rev. B 56, 7882 (1997).

[12] A. A. Abrikosov and L. P. Gor'kov, Zh. Eksp. Teor. Fiz. 39, 1781 (1960) [Sov. Phys. JETP 12, 1243 (1961)].

[13] Y. Dalichaouch, M. C. de Andrade, D. A. Gajewski, R. Chau, P. Visani, and M. B. Maple, Phys. Rev. Lett. 75, 3938 (1995).

[14] J. P. Carbotte, Rev. Mod. Phys. 62, 1027 (1990).

[15] R. J. Radtke, K. Levin, H.-B. Schüttler, and M. R. Norman, Phys. Rev. B 48, 653 (1993).

[16] M. Prohammer, A. Perez-Gonzalez, and J. P. Carbotte, Phys. Rev. B 47, 15152 (1993).

[17] M. El-Hagary, H. Michor, and G. Hilscher, Phys. Rev. B 61, 11695 (2000).

[18] E. Puchkaryov and K. Maki, Eur. Phys. J. B 4, 191 (1998).

[19] S. Skalski, O. Betbeder-Matibet, and P. R. Weiss, Phys. Rev. 136, A1500 (1964).

[20] P. J. Hirschfeld, P. Wölfle, and D. Einzel, Phys. Rev. B 37, 83 (1988).

[21] Y. Suzumura and H. J. Schulz, Phys. Rev. B 39, 11398 (1989).

[22] The authors of Ref. [6] normalize the angular function $\phi(\mathbf{n})$ by the condition $\left\langle\phi^{2}(\mathbf{n})\right\rangle_{F S}=1$. The authors of Refs. [18, 20] and [21] present the results for several specific functions $\phi(\mathbf{n})$ corresponding to different values of $\left\langle\phi^{2}(\mathbf{n})\right\rangle_{F S}$ and $\left\langle\phi^{4}(\mathbf{n})\right\rangle_{F S}$. The authors of Ref. [19] express their results in terms of the Riemann zeta functions $\zeta(n, z)=\sum_{k=0}^{\infty}(k+z)^{-n}$ which are related to the polygamma functions $\psi^{(n)}(z)$ by $\psi^{(n)}(z)=$ $(-1)^{n+1} n ! \zeta(n+1, z)$.

[23] T. M. Mishonov, E. S. Penev, and J. O. Indekeu, Phys. Rev. B 66, 066501 (2002); Europhys. Lett. 61, 577 (2003).

[24] T. M. Mishonov, E. S. Penev, J. O. Indekeu, and V. L. Pokrovsky, Phys. Rev. B 68, 104517 (2003).

[25] P. W. Anderson, J. Phys. Chem. Solids 11, 26 (1959).

[26] A. A. Abrikosov, Physica C 214, 107 (1993). 
[27] F. Pesty, K. Wang, and P. Garoche, Synth. Met. 27, B137 (1988).

[28] E.-W. Scheidt, T. Schreiner, P. Kumar, and G. R. Stewart, Phys. Rev. B 58, 15153 (1998).

[29] J. W. Loram, K. A. Mirza, and P. F. Freeman, Physica C 171, 243 (1990).

[30] C. Meingast, R. Ahrens, B. Blank, H. Bürkle, B. Rudolf, and H. Wühl, Physica C 173, 309 (1991).

[31] S. Shamoto, T. Kiyokura, H. Harashina, and M. Sato, J. Phys. Soc. Jap. 63, 2324 (1994).

[32] S. Kim, R. A. Fisher, N. E. Phillips, and J. E. Gordon, Physica C 162-164, 494 (1989).

[33] K. A. Mirza, J. W. Loram, and J. R. Cooper, Physica C 282-287, 1411 (1997).

[34] L. A. Openov, submitted to Phys. Rev. Lett.

[35] F. Rullier-Albenque, H. Alloul, and R. Tourbot, Phys. Rev. Lett. 91, 047001 (2003). 


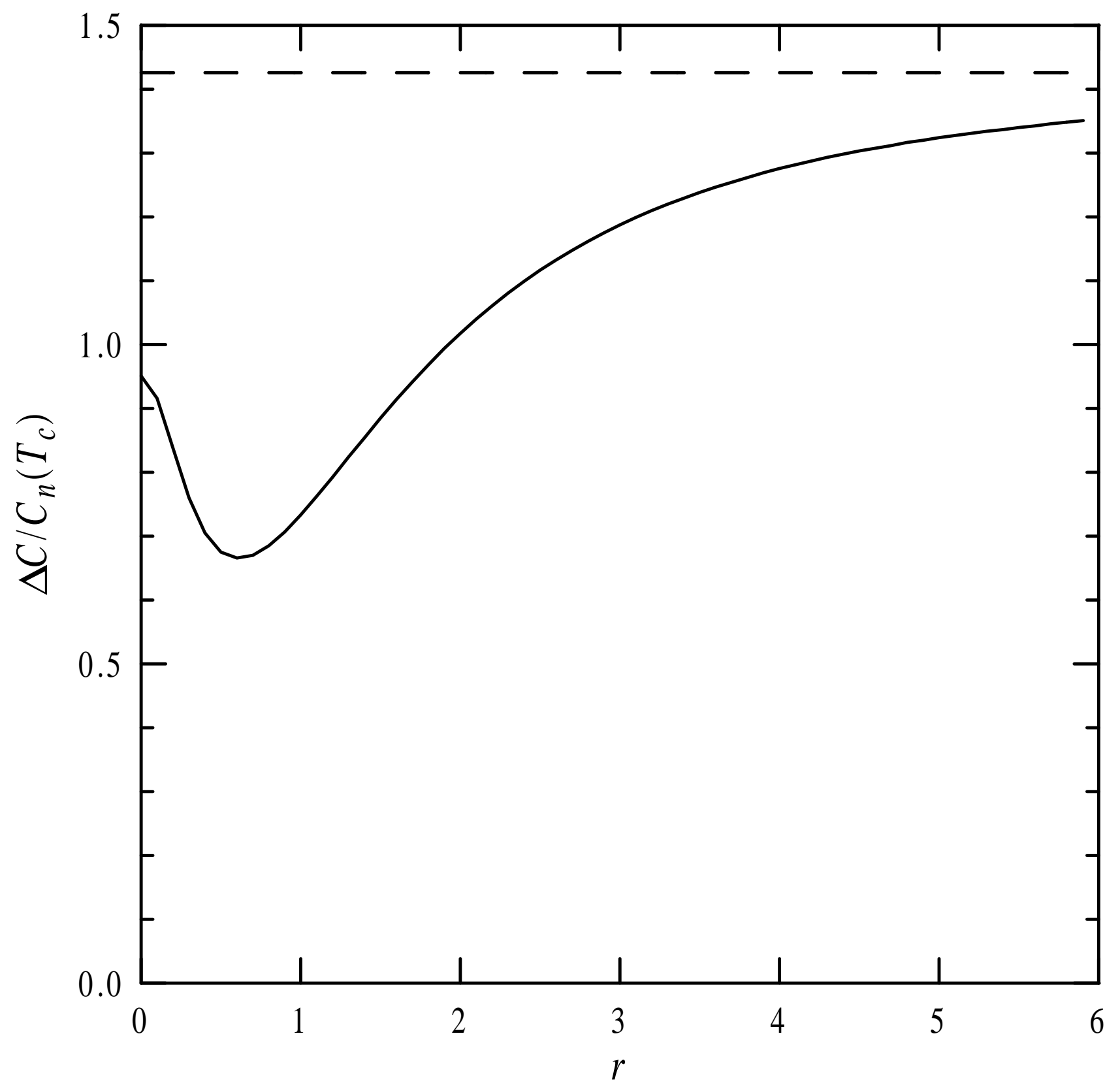

Fig. 1. Specific heat jump $\Delta C$ normalized by the normal state specific heat $C_{n}\left(T_{c 0}\right)$ versus the coefficient $r$ that specifies the anisotropy of the superconducting order parameter $\Delta(\mathbf{p})=\Delta[r+\cos (2 \varphi)]$, for a clean superconductor without any impurities. Dashed line shows the value of $\Delta C / C_{n}\left(T_{c 0}\right)=12 / 7 \zeta(3) \approx 1.426$ in an isotropic s-wave superconductor. 


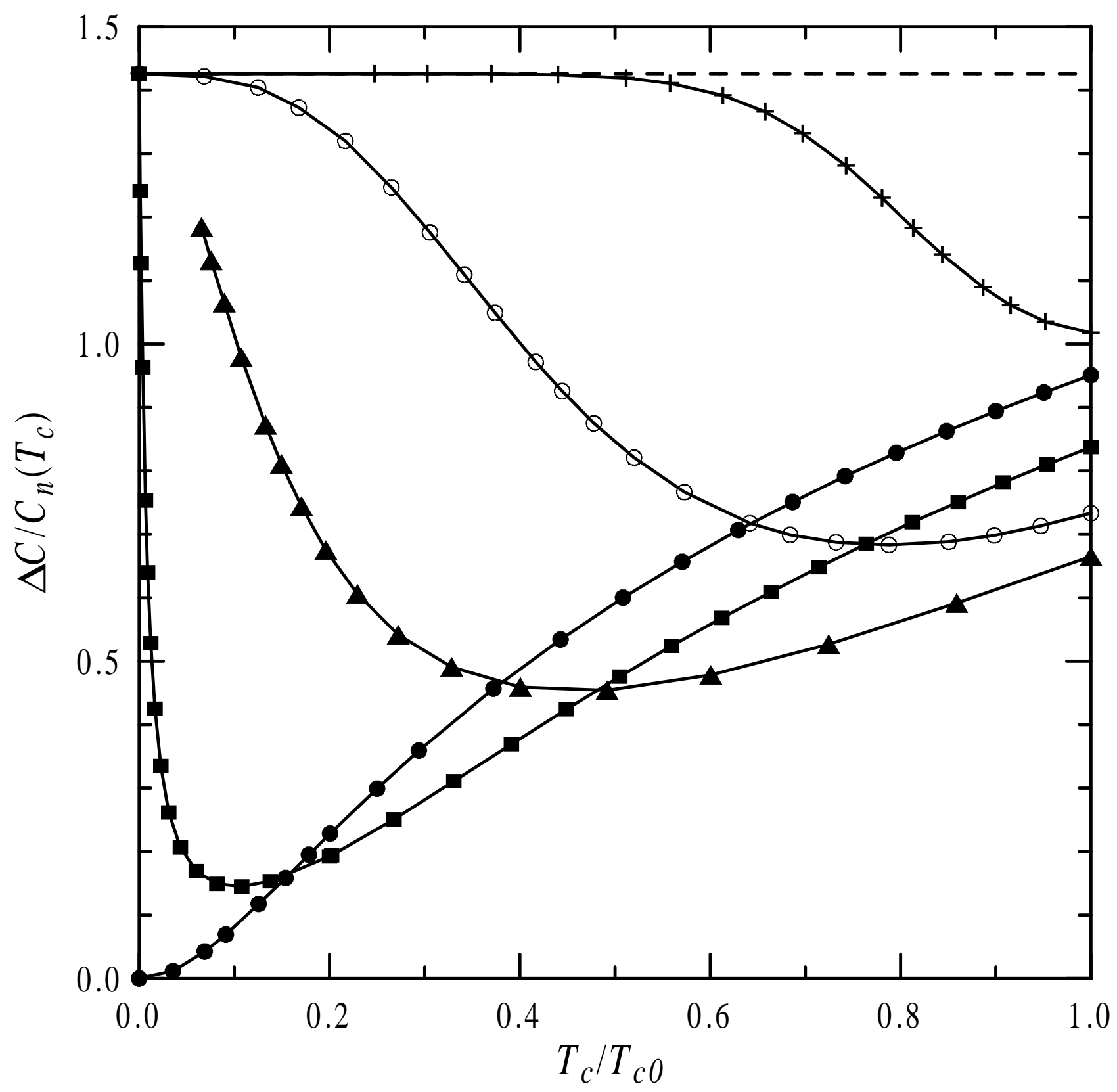

Fig. 2. Specific heat jump $\Delta C$ normalized by the normal state specific heat $C_{n}\left(T_{c}\right)$ versus the normalized critical temperature $T_{c} / T_{c 0}$ for a superconductor disordered by nonmagnetic impurities. The superconducting order parameter is assumed to have the form $\Delta(\mathbf{p})=\Delta[r+\cos (2 \varphi)]$, where $r=0$ (closed circles); 0.2 (squares); 0.6 (triangles); 1 (open circles); 2 (pluses). Solid lines are guides for the eye. Dashed line corresponds to an isotropic s-wave $(r \rightarrow \infty, \Delta r \rightarrow$ const $)$. 


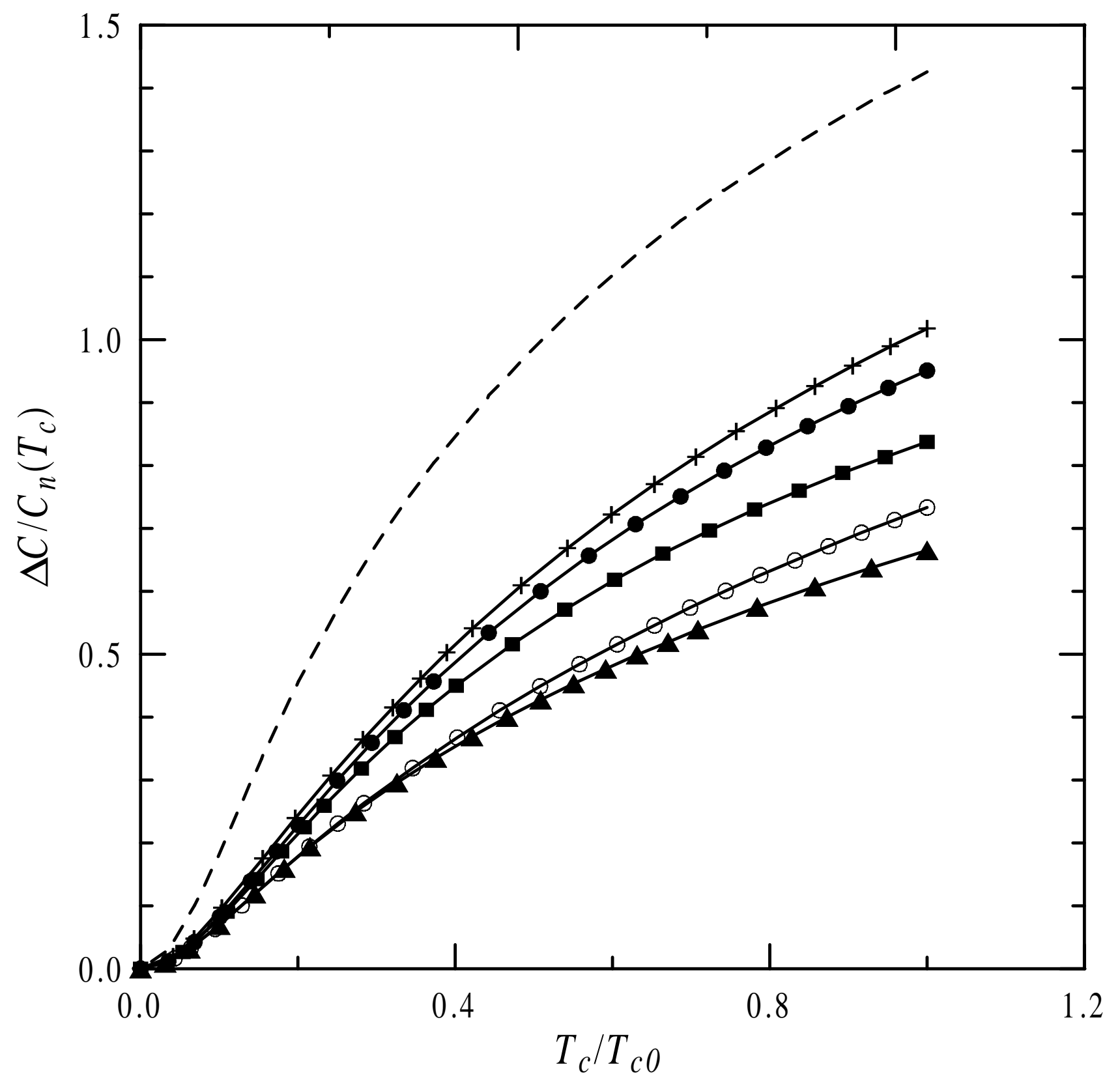

Fig. 3. Same as in Fig. 2 for a superconductor disordered by spin-flip scatterers only. 


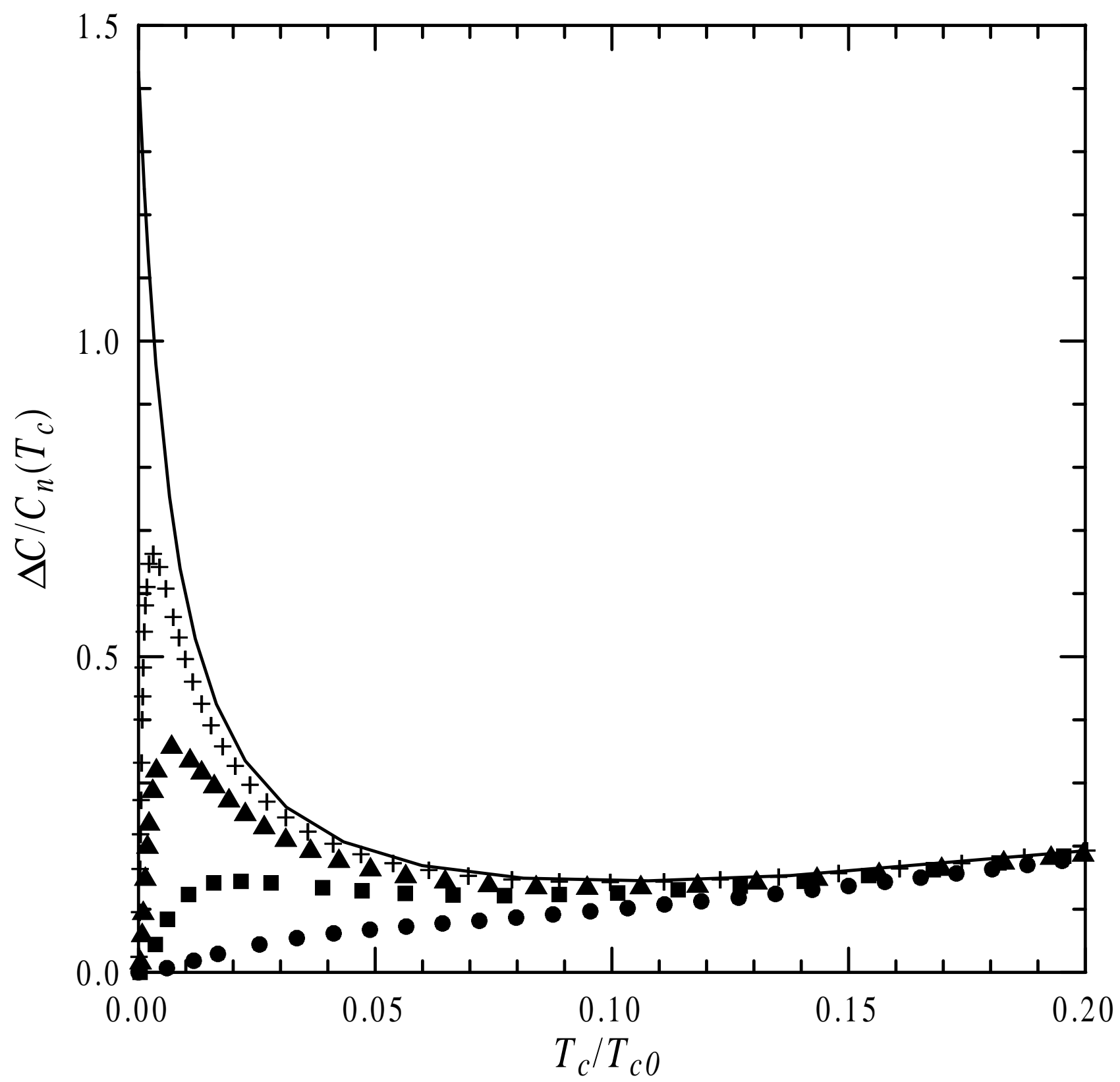

Fig. 4a. Specific heat jump $\Delta C$ normalized by the normal state specific heat $C_{n}\left(T_{c}\right)$ versus the normalized critical temperature $T_{c} / T_{c 0}$ for a superconductor with the order parameter $\Delta(\mathbf{p})=\Delta[r+\cos (2 \varphi)]$. The superconductor initially contains a small amount of spin-flip scatterers and thus has the initial critical temperature $T_{c 0}^{\prime}<T_{c 0}$. It is further disordered by nonmagnetic impurities only, so that the concentration of potential scatterers exceeds that of spin-flip scatterers. $r=0.2 . \delta T_{c o} / T_{c 0}=\left(T_{c 0}-T_{c 0}^{\prime}\right) / T_{c 0}=0$ (solid line), 0.001 (pluses), 0.003 (triangles), 0.01 (squares), and 0.03 (circles). These values of $\delta T_{c o} / T_{c 0}$ correspond to the values of the spin-flip pair breaking rate $\rho_{s 0}=1 / 2 \pi \tau_{s} T_{c 0}=0,0.00038,0.00113,0.00376$, and 0.01127 respectively. Note that at $T_{c} / T_{c 0}>0.2$ the curves of $\Delta C / C_{n}\left(T_{c}\right)$ versus $T_{c} / T_{c 0}$ for different $\rho_{s 0}$ (i. e., for different $\delta T_{c o}$ ) almost coincide. 


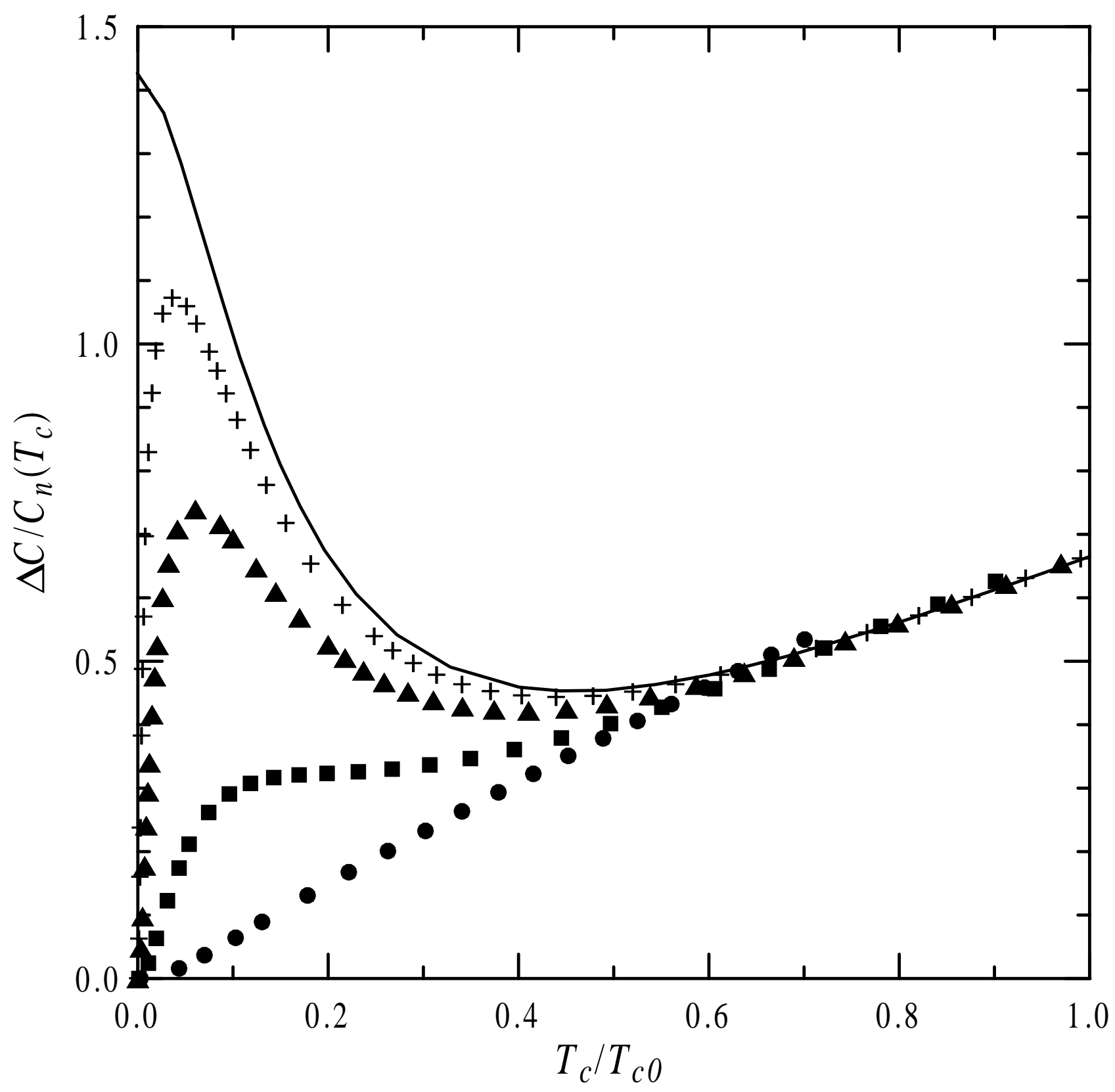

Fig. 4b. Specific heat jump $\Delta C$ normalized by the normal state specific heat $C_{n}\left(T_{c}\right)$ versus the normalized critical temperature $T_{c} / T_{c 0}$ for a superconductor with the order parameter $\Delta(\mathbf{p})=\Delta[r+\cos (2 \varphi)]$. The superconductor initially contains a small amount of spin-flip scatterers and thus has the initial critical temperature $T_{c 0}^{\prime}<T_{c 0}$. It is further disordered by nonmagnetic impurities only, so that the concentration of potential scatterers exceeds that of spin-flip scatterers. $r=0.6 . \delta T_{c o} / T_{c 0}=0$ (solid line), 0.01 (pluses), 0.03 (triangles), 0.1 (squares), and 0.3 (circles). [ $\rho_{s 0}=0,0.00285,0.00854,0.02823$, and 0.08232 respectively]. 


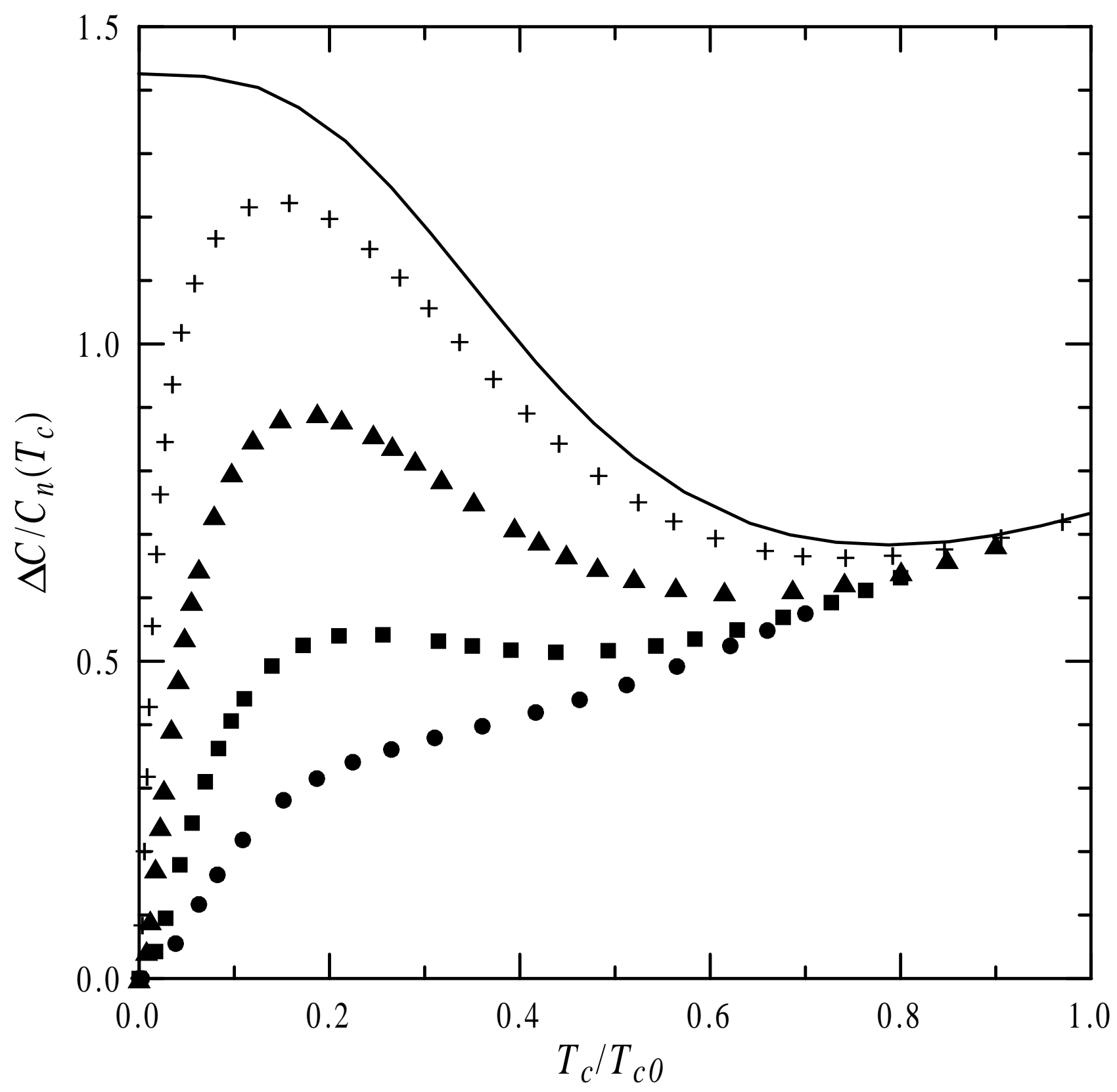

Fig. 4c. Specific heat jump $\Delta C$ normalized by the normal state specific heat $C_{n}\left(T_{c}\right)$ versus the normalized critical temperature $T_{c} / T_{c 0}$ for a superconductor with the order parameter $\Delta(\mathbf{p})=\Delta[r+\cos (2 \varphi)]$. The superconductor initially contains a small amount of spin-flip scatterers and thus has the initial critical temperature $T_{c 0}^{\prime}<T_{c 0}$. It is further disordered by nonmagnetic impurities only, so that the concentration of potential scatterers exceeds that of spin-flip scatterers. $r=1 . \delta T_{c o} / T_{c 0}=0$ (solid line), 0.03 (pluses), 0.1 (triangles), 0.2 (squares), and 0.3 (circles). [ $\rho_{s 0}=0,0.00727,0.02400,0.04728$, and 0.06975 respectively]. 


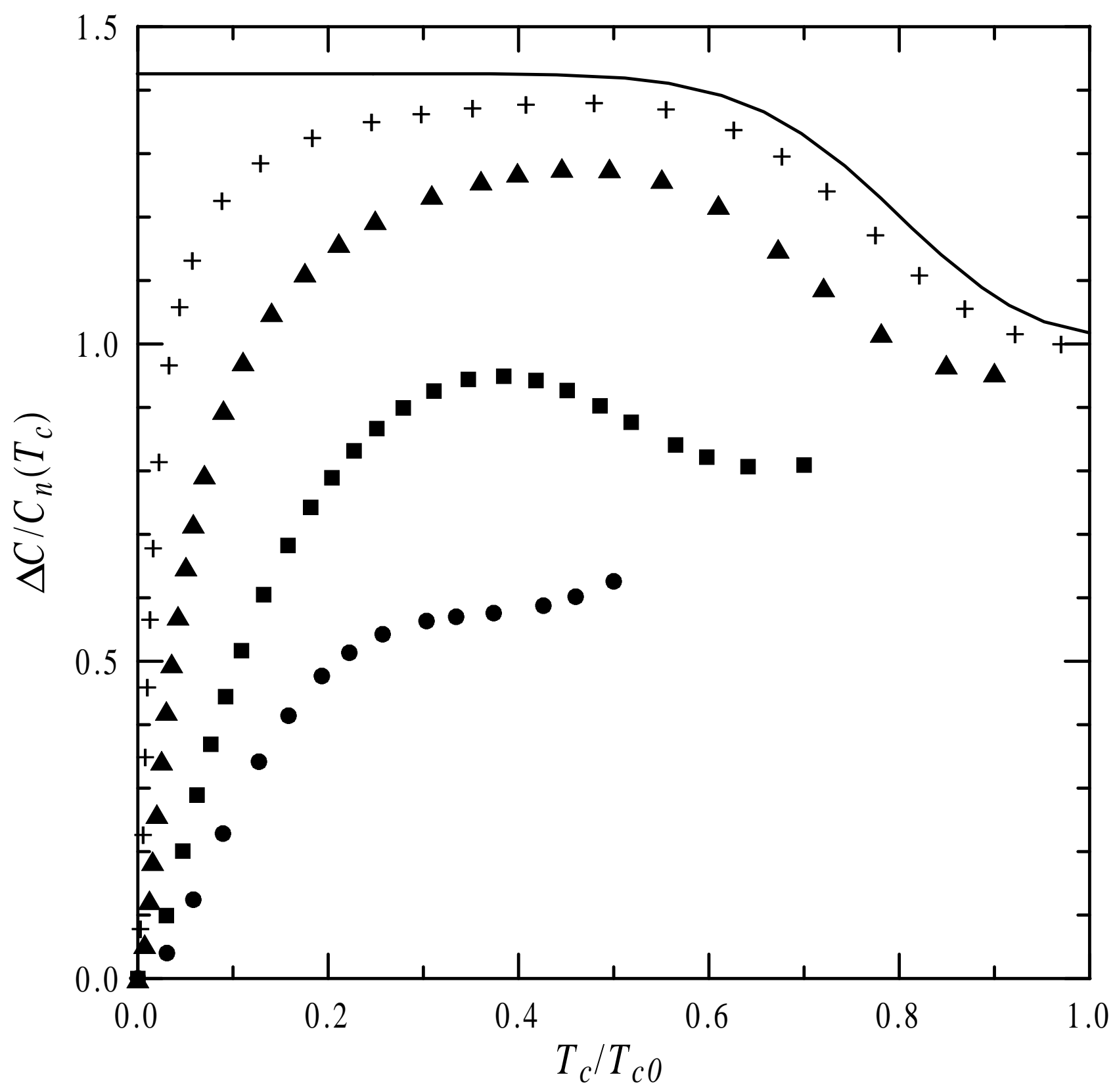

Fig. 4 d. Specific heat jump $\Delta C$ normalized by the normal state specific heat $C_{n}\left(T_{c}\right)$ versus the normalized critical temperature $T_{c} / T_{c 0}$ for a superconductor with the order parameter $\Delta(\mathbf{p})=\Delta[r+\cos (2 \varphi)]$. The superconductor initially contains a small amount of spin-flip scatterers and thus has the initial critical temperature $T_{c 0}^{\prime}<T_{c 0}$. It is further disordered by nonmagnetic impurities only, so that the concentration of potential scatterers exceeds that of spin-flip scatterers. (d) $r=2 . \delta T_{c o} / T_{c 0}=0$ (solid line), 0.03 (pluses), 0.1 (triangles), 0.3 (squares), and 0.5 (circles). [ $\rho_{s 0}=0,0.00641,0.02114,0.06116$, and 0.09731 respectively]. 


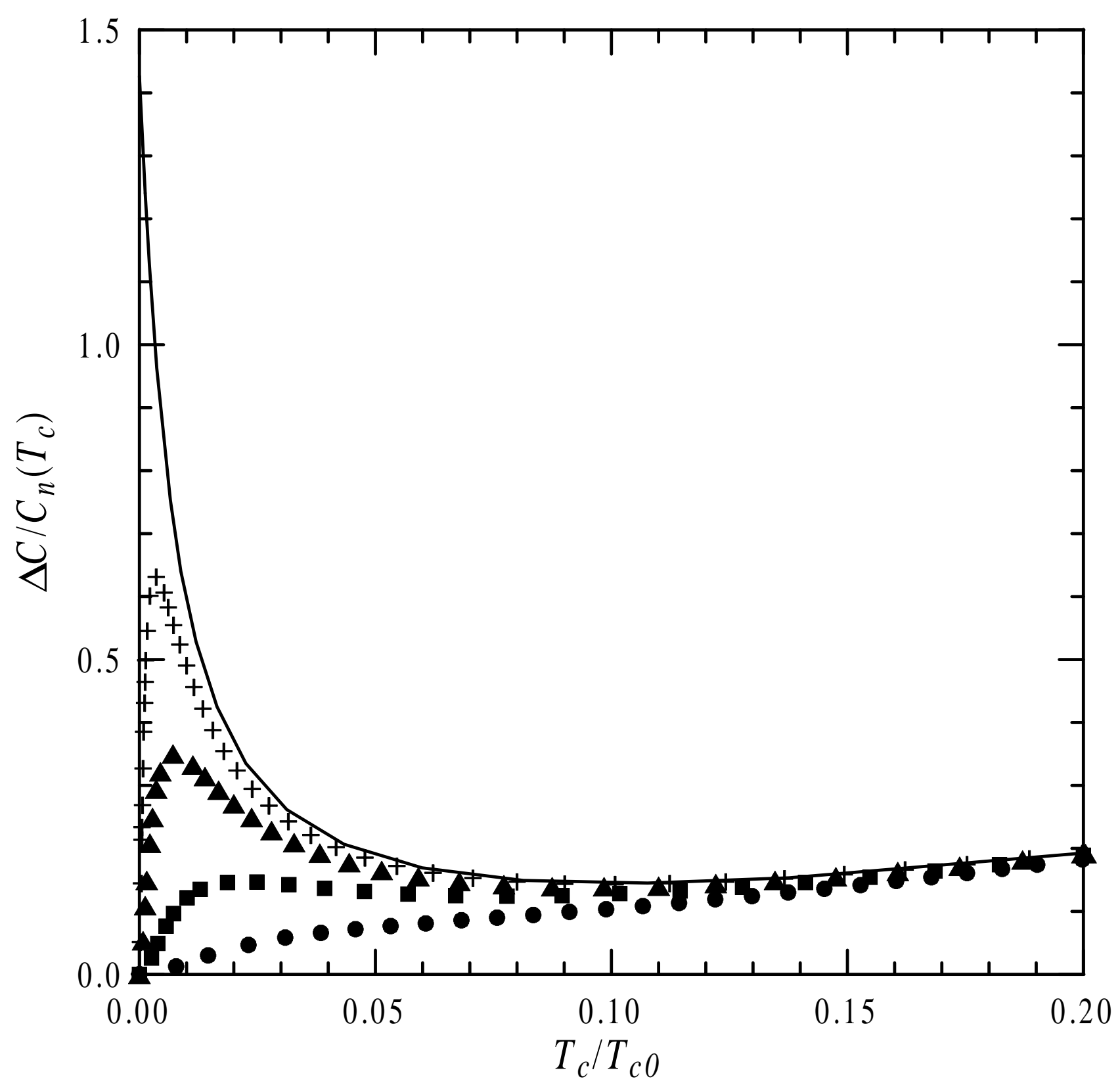

Fig. 5a. Same as in Fig. 4 for a superconductor with a constant ratio $\alpha$ of spin-flip to potential scattering rates. $r=0.2 . \alpha=0$ (solid line), 0.001 (pluses), 0.003 (triangles), 0.01 (squares), and 0.03 (circles). Note that at $T_{c} / T_{c 0}>0.2$ the curves of $\Delta C / C_{n}\left(T_{c}\right)$ versus $T_{c} / T_{c 0}$ for different $\alpha$ almost coincide. 


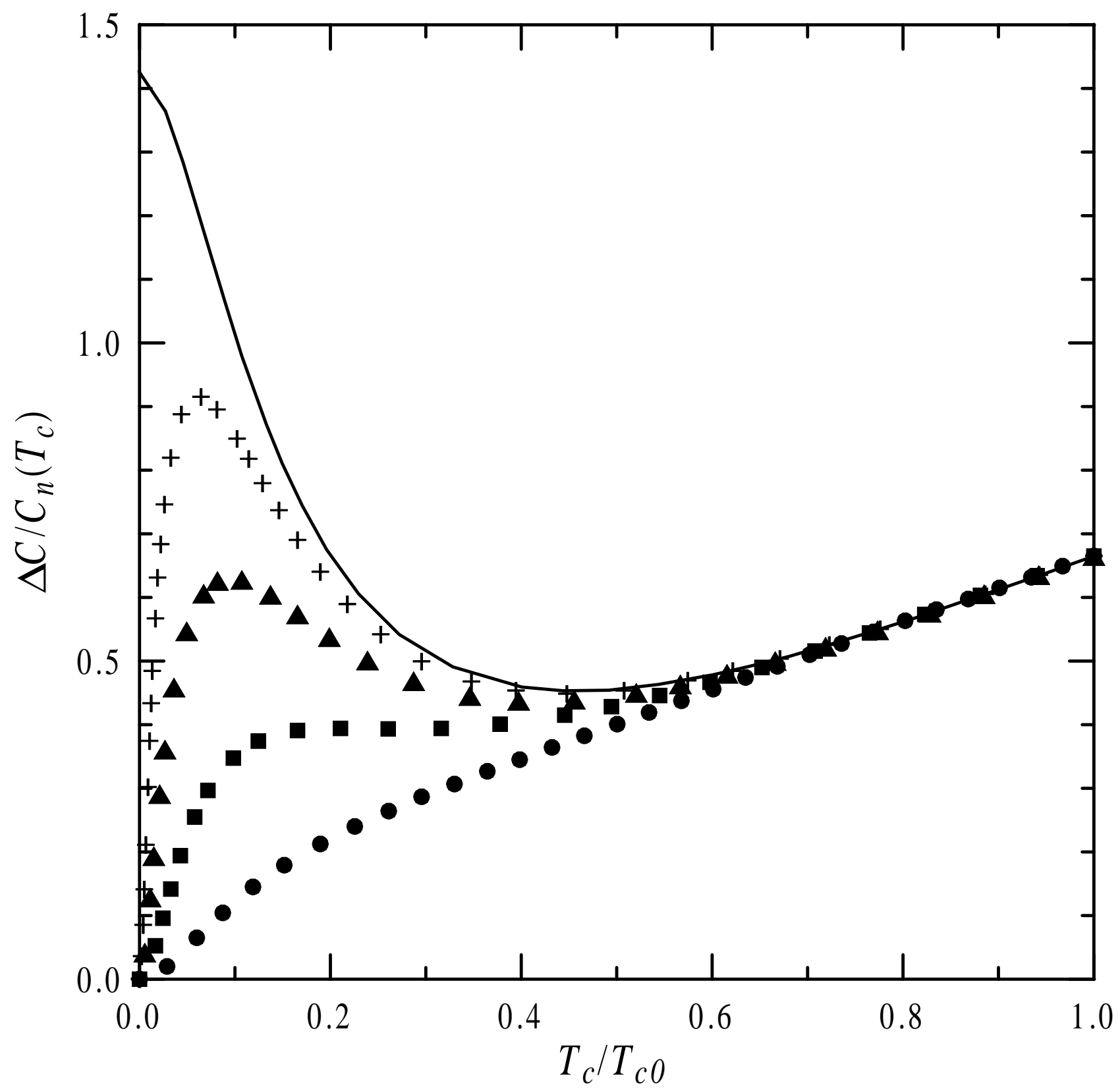

Fig. 5b. Same as in Fig. 4 for a superconductor with a constant ratio $\alpha$ of spin-flip to potential scattering rates. $r=0.6 . \alpha=0$ (solid line), 0.003 (pluses), 0.01 (triangles), 0.03 (squares), and 0.1 (circles). 


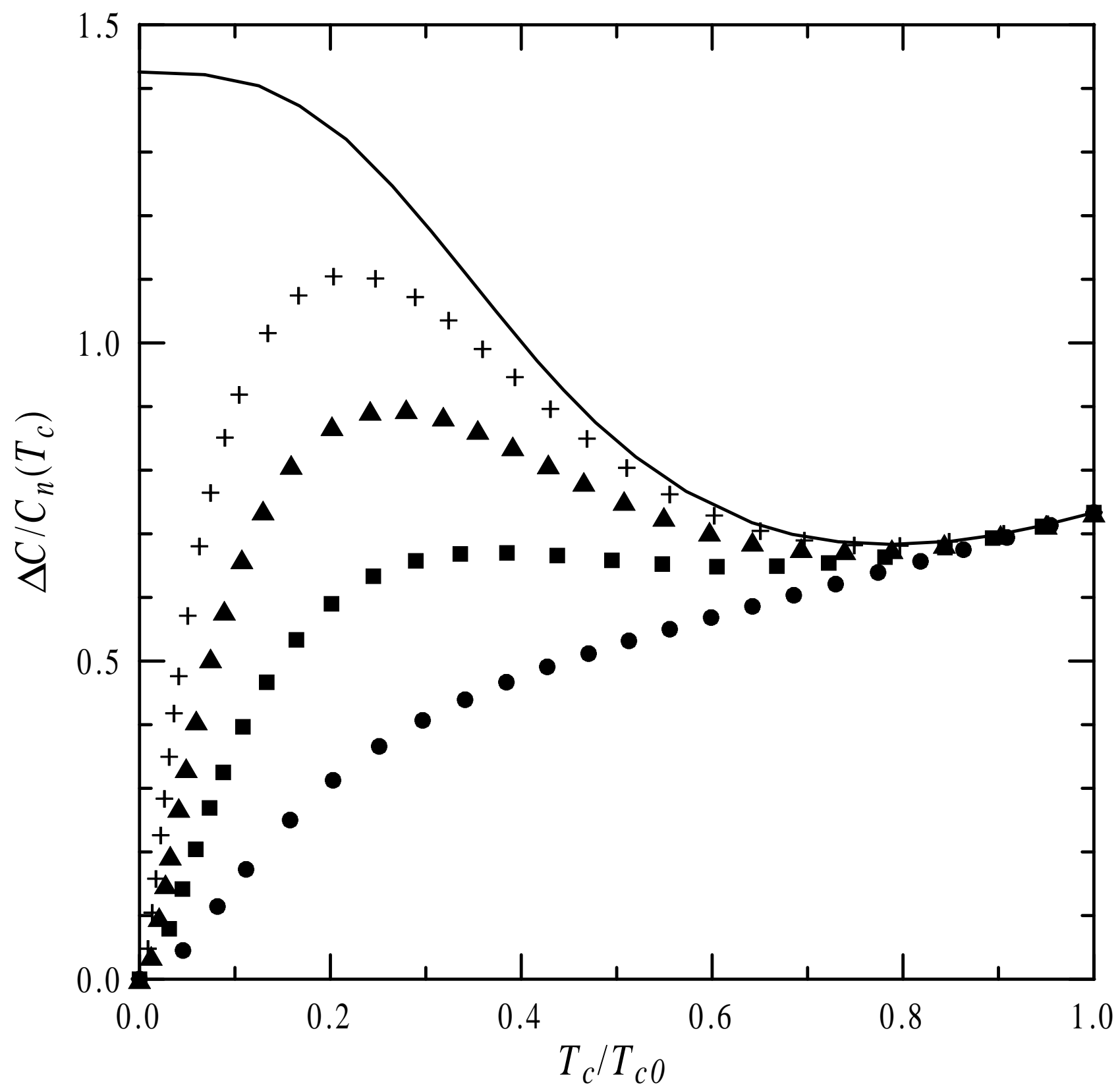

Fig. 5c. Same as in Fig. 4 for a superconductor with a constant ratio $\alpha$ of spin-flip to potential scattering rates. $r=1 . \alpha=0$ (solid line), 0.003 (pluses), 0.01 (triangles), 0.03 (squares), and 0.1 (circles). 


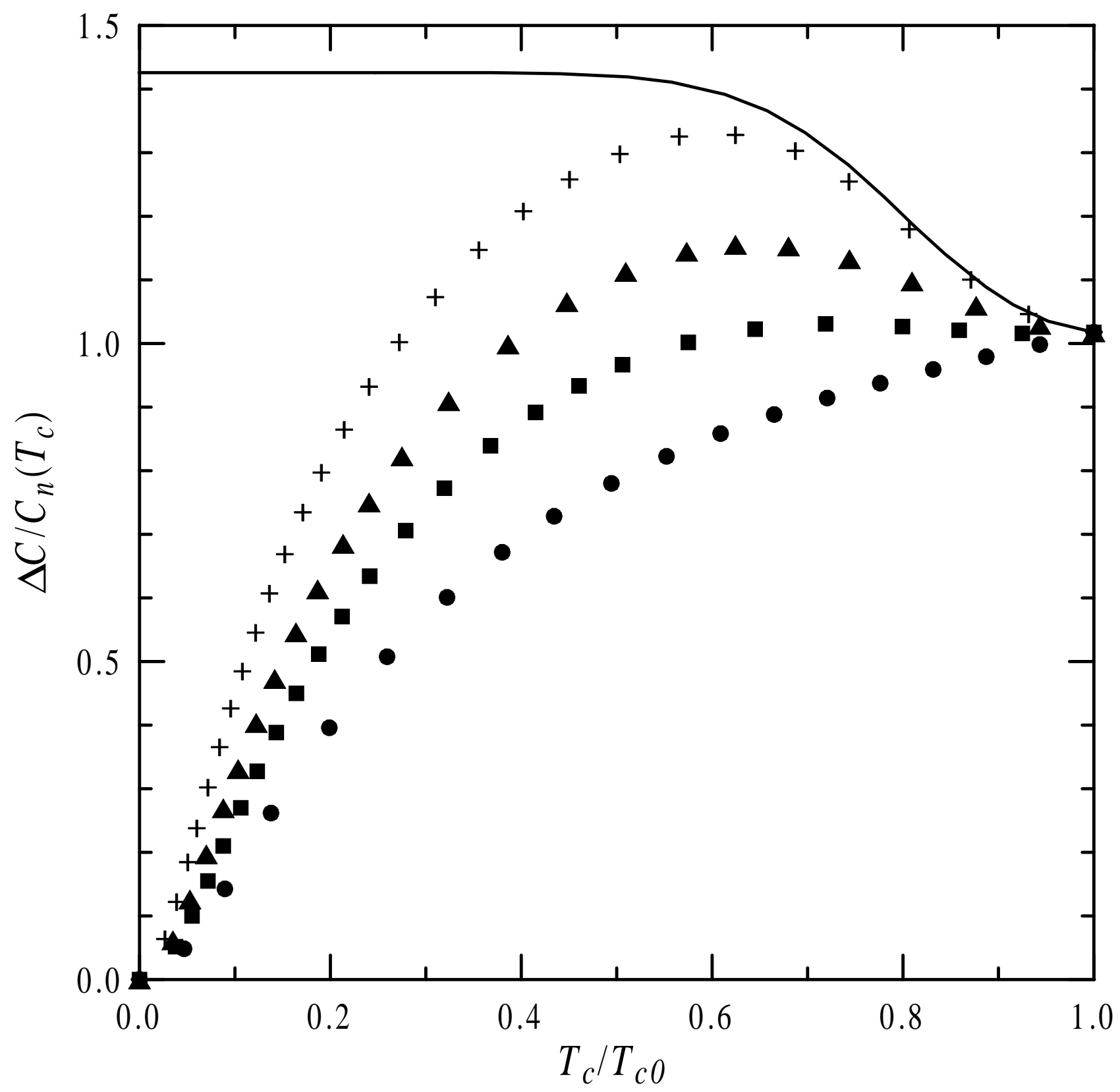

Fig. 5d. Same as in Fig. 4 for a superconductor with a constant ratio $\alpha$ of spin-flip to potential scattering rates. $r=2 . \alpha=0$ (solid line), 0.001 (pluses), 0.01 (triangles), 0.03 (squares), and 0.1 (circles). 